and cyanosis; dyspnca was still present. At no time has clubbing of the fingers been noticed. During the past winter the patient has been under medical care at Dresden and effervescent carbon-dioxide baths and moderate exercises were prescribed. On Feb. 3rd of the present year the patient was again seen by us. He is quite free from all dyspnoea even on great exertion, and he has completely regained his weight. The condition of the chest is but little aitered from what was observed on June 29th, 1907, the difference being that the dulness previously observed is now a little lower and the upper right chest is a little more resonant than normal. The obscurity of the cardiac dulness is maintained.

Radiographic examination. - This was carried out by Mr. R. Higham Cooper on March 3rd, 1908. The shadow shown by the heart was normal in extent and position, and the diaphragm reached to the level of the fifth rib in the nipple line on both sides during expiration; posteriorly during expiration the dome of the diaphragm forming the upper limit of the liver reached as high as the seventh rib in the scapular line.

Comments.-In reviewing the illness and the subsequent developments it appears to us that on May 2nd, 1907, an embolus was dislodged from one of the veins of the lower extremities or a thrombus ${ }^{1}$ was dislodged from the pulmonary artery and was driven into the lower division of the right pulmonary artery-i.e., into the branch supplying the middle and lower lobes of the right lung. These two lobes became atelectatic and the space formerly occupied by them when fully expanded was filled up by the ascent of the liver and right half of the diaphragm, and also probably by compensatory emphysema of the left lung in the direction of the right chest, accompanied by the upward dislocation of the stomach and left half of the diaphragm. The area of cardiac dulness was, especially towards the left, obscured by the compensatory emphysema of the left lung, the heart itself being little altered, if at all, so far as clinical examination could determine, in its normal position. We believe that the upper lobe of the right lung is undergoing compensatory hypertrophy and we anticipate that in the course of time it will be able completely to occupy the space formerly filled by the three lobes of the right lung if it has not already almost done so, as the diaphragm as shown by the $x$ rays now during expiration reaches the height of the fifth rib in the right nipple line, the level reached in a normal individual, though probably the normal level in our patient owing to his original emphysema is lower. This view is supported by the observation by one of us that in a patient who died from an acute illness the left lung consisted almost entirely of the upper lobe of the definitive lung, the lower one being represented by an attenuated mass of atelectatic lung substance with a complete but small system of bronchial tubes, the whole not being much larger than a hen's egg.

A blood examination made 19 days after the accident of May 2nd, 1907, showed 5,687,500 red corpuscles and 9375 white corpuscles per cubic millimetre; the relative proportions of the latter were normal ; the hæmoglobin amounted to more than 100 per cent., from which it may be surmised that the hæmoglobin had increased beyond the normal limits in response to a reduction of the normal lung substance?

We are unable to find any reference to any account of surviral after such an extensive obliteration of lung substance by an embolus or thrombus. Cohn refers in the section of his work ${ }^{2}$ dealing with embolus of the pulmonary artery to two cases (Cases 1 and 14) in which at the post-mortem exami nation old or partly old clots were found in the pulmonary artery, but there is no information which would lead us to understand that the actual deposit of the embolus had taken place and had been clinically observed at a time as long as 11 months previously, as in our case. Our case now shows no signs of distress in breathing and in fact he is quite well. We believe that the reason why in this case the heart has not been dislocated into the right chest, as happens so often in tuberculosis and fibrosis of the right lung, is to be found in the possibility that the diaphragm, unaltered and unhampered by inflammation as it was, was readily able to yield and so allow the liver and the left lung instead of the

1 Tide paper by Dr. C. R. Box, Transactions of the Clinical Society of London, 1906, p. 189. Klinik der Embolischen Gefässkrankheiten, Berlin, 1860. heart to fill the place of the collapsed lung, or it may be that the mediastinal structures in our patient are more firm and less capable of actual displacement.

\section{NOTES ON THE THERAPEUTIC APPLICA- TION OF STOCK VACCINES IN THE TREATMENT OF BACTERIAL INFECTIONS.}

BY JOHN MATTHEWS, B.A., B.C. CANTAB., ASSISTANT IN THE DEPARTMENT OF THERAPEUTIC INOCULATION, ST. MARY'S HOSPITAL, LONDON.

OPsonINs is the name given by Wright and Douglas to those protective substances in the blood serum in the absence of which leucocytes are only capable of phagocytosis in a very limited degree. As immunity to bacterial infections depends largely on phagocytosis opsonins play an extremely important part in producing immunity. It has been shown that after recovering from a microbic infection the proportion of protective substances, or opsonins, in the patient's serum connected with that particular infection is higher, to a greater or less extent, than in the serum of a normal healthy individual. It has also been shown that if an inoculation of killed microbes be made into a normal individual the proportion of opsonins in his serum will rise as compared with the serum of a normal individual not so inoculated.

In the vast majority of microbic infections the physiological mechanism of immunity reacts so quickly to the infection that an adequate supply of protective substances is brought to the site of infection and the phagocytes are enabled to cope with the microbes. Such an infection will rarely be attended with symptoms either objective or subjective, and will therefore be unnoticed. In cases which are not so aborted and which therefore become noticeable as local or general infections, either the infection has been too virulent for the normal mechanism to cope with or for some reason, such, for example, as great exhaustion due to exposure or local injury, the normal mechanism for the production of immunity is deficient. Therapeutic inoculation supplies a method of artificially producing immunity and the object of this paper is to indicate the potentialities and limitations of therapeutic inoculation in the treatment of microbic infections, generally with the assistance given by the frequent estimation of the opsonic index but more especially without the aid derived from the opsonic index.

The opsonic index is the expression of the measure of the phagocytic power produced in a sample of washed leucocytes by the serum of the individual in question, as compared with the phagocytic power induced in a similar sample of leucocytes by the serum of one or more healthy individuals. This latter power is taken as unity, and the index of an infected individual is expressed as a fraction of unity, above or below unity according to the greater or less quantity of protective substances in the infected serum, as compared with the normal serum. The opsonic index then is the measure of the resisting power, as comprised in phagocytosis, of a serum, against the microbe in question.

A vacoine is a killed bacterial culture suspended in sterilised normal saline solution, to which lysol to the strength of $\frac{1}{4}$ per cent. or carbolic acid to the strength of $\frac{1}{2}$ per cent. has been added. Vaccines are accurately standardised by mixing together definite quantities of vaccine and normal human blood of known corpuscular content. Smears are made of the mixture, are dried, fixed and stained, and the numbers of red cells and microbes in many successive fields of the microscope are counted. An estimation of the number of microbes in each cubic centimetre of vaccine is then possible. The cultures are killed by immersion for one hour in a water bath at $60^{\circ} \mathrm{C}$., and the sterility having been demonstrated by culture methods a suitable dilution for use is made in rubber-capped sterile bottles for laboratory and hospital use, and in sealed glass sterilised capsules, containing slightly more than one cubic centimetre, for general use.

The effect of vaccines on the blood. - The injection of a dose of vaccine into a healthy normal individual produces either (1) an immediate rise in the protective substances, as indicated by the opsonic index; (2) a fall of longer or shorter duration, followed by a rise up to or above normal, in 
its turn followed by a fall towards normal ; or (3) a fall tending to be more or less permanent according as to whether the dose be small, medium, or excessive. These effects are diagrammatically represented in the appended charts.

The rise in the first case will, though quick, be short lived. In the second case the rise subsequent to the initial fall, or negative phase, will be more prolonged, and having reached its maximum will gradually fall towards the level it was at previous to inoculation. In the third case the fall may be so severe as to last a considerable time, in some cases even never regaining its previous level without a further inoculation of minimal doses of vaccine. All gradations between will occur according to the dose of vaccine, and these varying results are designedly aimed at, according to the nature of the case in hand. There is thus no ideal opsonic curve in general to be aimed at, though in a particular case one particular form of curve may be ideal.

The fall subsequent to inoculation is called by Wright the negative phase, the subsequent rise he calls the flow, and the gradual fall towards normal he calls the ebb, the tides of flow and ebb together constituting the positive phase. In addition a phenomenon has been noted, occurring mainly in auto-inoculations produced by passive congestion and similar measures called the false rise. Here a slight rise precedes the negative phase but it is of small importance. question of dosage. In the case of (1) this in certain conditions would be an ideal curve in the circumstances Taking, for example, a case of septicæmia, with or without a localised focus, it would be disastrous to induce a negative phase of any duration at all. During a negative phase of only a few hours' duration all the symptoms might be profoundly aggravated, the toxæmia deepened, the temperature raised and pain, if present, increased, and it is probable that owing to the low protective power on the part of the blood foci of suppuration would be initiated in many hitherto uninfected areas. Obviously in such cases the aim of the immunisator should be to produce a maximum rise in protective substances compatible with an entire absence of negative phase. Nor even if no negative phase be produced should the dose be large enough to delay the elaboration of protective sub. stances. If the dose be of such a size that the above desideratum be fulfilled the temperature should fall, pain and discharge should decrease, and a diminution of any local congestion and swelling that might be present should be noted, and these symptoms would in most cases be sufficiently marked to enable a determination as to re-inoculation to be made. Such cases as the above will usually be comprised in the following-erysipelas, cellulitis, lymphangitis, and puerperal fevers and septicæmias generally, in which, even if the process be more or less localised, a temporary lowering of resistance may be answerable for an alarming increase in the area of the process.

FIG. 1.

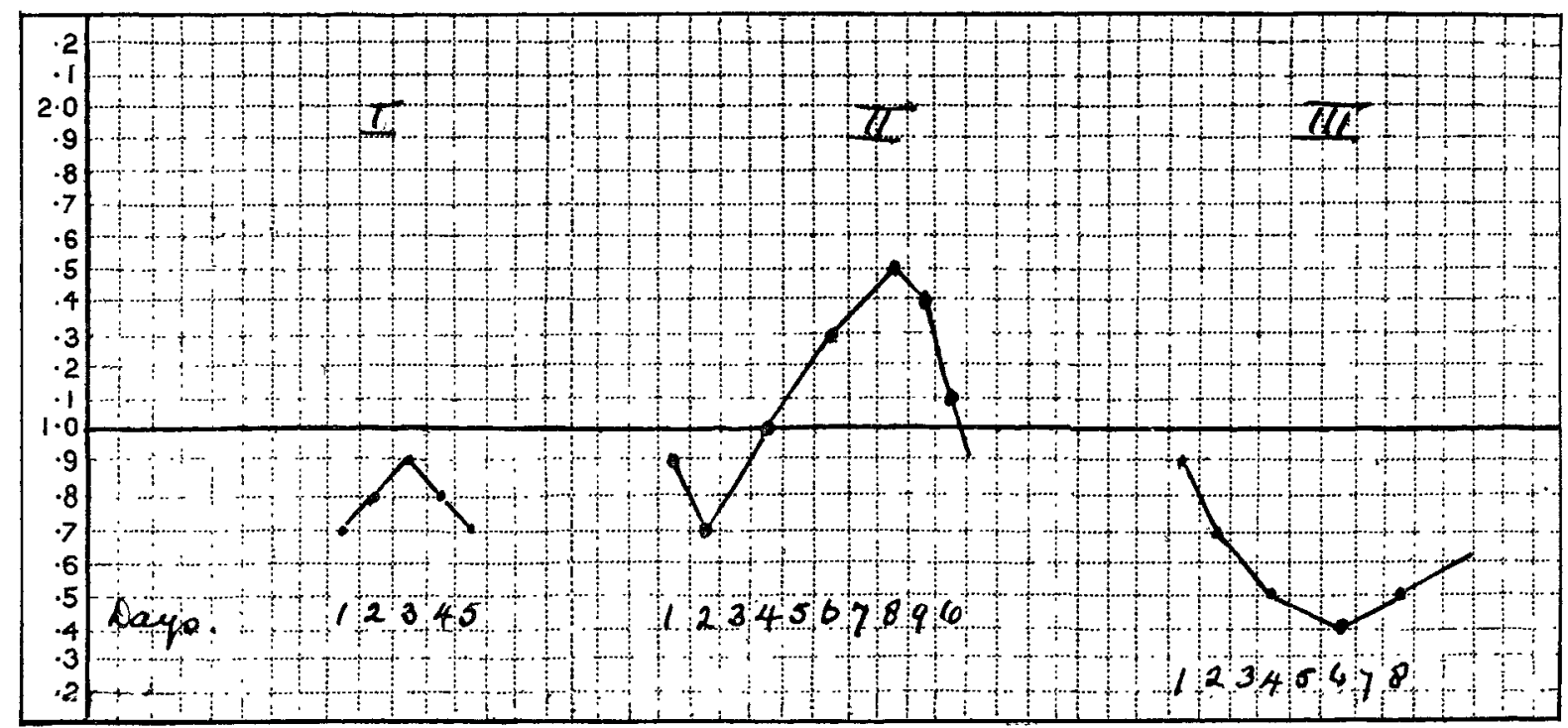

Diagramatic opsonic charts of the effects achieved by varying doses of vaccine. Referred to in the text as Diagrams (1), (2), and (3).

In general in the treatment of infections it is desirable to maintain an average high level of resistance. Thus, in the case of an effect such as Diagram 1 re-inoculation would be necessary very shortly; in Diagram 2 a second inoculation would be indicated, as the curve was falling to normal; whereas a minimal dose of vaccine might be advisable in Diagram 3 when the fall had ceased to bring the resistance back to the initial position.

Within limits it may be said that the duration of the effects produced by any one inoculation varies proportionately to the dose of vaccine inoculated-that is to say, that the longer the negative phase the longer the positive phase will be. However, a point is soon reached beyond which the duration of the negative phase is only increased at the expense of the positive phase, and ultimately no positive phase accrues at all, as indicated in Diagram 3. Here it may be stated that no attempt is made, except in cases of very low resistance initially, to superpose one positive phase upon another without any intervening negative phase. In general, an effect similar to that depicted in Diagram 2 is aimed at, constantly repeated, so that the sum total of the individual effects of a series of inoculations shall result in longer average duration of time of increased immunity and shorter average duration of time of decreased immunity as compared with that of a normal individual.

Before leaving the consideration of the three classes of curves produced by varying doses of vaccine it may be advisable to correlate with the opsonic results certain clinical symptoms and at the same time consider generally the
Thus it follows that in any infection which is not strictly localised, and more especially in septicæmias, the curve to be aimed at is one in which no negative phase occurs. In other words, the dose must be minimal in size. The corollary is that the response invoked is short, and therefore reinoculation must quickly follow, even to daily doses. In such cases the opsonic index is of paramount value as a guide, but as indicated above much assistance as to dosage and interspacing may be gained by the general symptoms and local signs.

Apart, however, from such severe cases as have been taken into consideration above, where a temporary lowering of the resistance is absolutely disastrous, it is inadvisable to give such a dose as to cause a negative phase in many chronic local conditions of less severity. To illustrate my meaning I will instance such a case as that of chronic tuberculous lymphatic glands. Such a case might well, and does mostly, show a constant low resisting power, as indicated by several successive tuberculo-opsonic indices of between 0.70 and $0 \cdot 80$. In such a case it would be desirable to aim in the first instance at such a curve as that in Diagram 1, so that a resistance equal to normal might be achieved without any intervening negative phase. A normal index having been thus attained either by one or two minimal doses of tuberculin, larger doses might then be given; an effect being aimed at such as depicted in Diagram 2, which will shortly come under consideration.

In considering those cases in which it is desirable to aim at producing the effect depicted in Diagram 2 we encounter 
a much larger class of case than we considered in connexion with Diagram 1. 'I'he majority of cases which the immunisator is called upon to treat are comprised in chronic or acute, strictly localised processes. In some the resistance of the circulating blood is actually above normal ; in most it is, however, below normal, and is likely to be variable. In other words, the state of resistance of the circulating blood may not necessarily have an immediate effect upon the local infection. Hence, a temporary negative phase, if followed by a more or less prolonged positive phase, will be of no consequence. The practical result is that by giving such a dose that a small negative phase is produced we obtain a positive phase that will probably last seven or eight days, and so the injections may be interspaced by a period of some 10 or 12, or even more, days. In this class of case we may therefore consider the result depicted in Diagram 2, an " ideal curve."

In most cases the negative phase should not last longer than 24 hours. The temperature will be no guide, for most of such cases will be apyrexial, and the indications as to dosage, apart from opsonic indices, will be mainly local. They will be objective and subjective. The subjective symptoms will be some or all of the following: malaise and increased tenderness and pain at the site of the lesion and in bladder cases increased frequency of micturition. Objectively there will be some or all of the following: increase of discharge, swelling, and congestion, and in the case of acne, and such like more or less generalised local infections, an increase in the number of foci of infection, and in bladder cases perhaps more cloudiness due to increased activity of the infective microbe. In short, during a negative phase an increase of those signs and symptoms due to the infection under consideration will be noticed. Such increase should only be noticeable for not more than 24 hours, and subsequently an amelioration should take place.

It is hardly necessary to point out that should any of the symptoms above mentioned be severe it would be advisable to return to smaller doses-that is to say, an endeavour should be made to proceed as in those cases in which Diagram 1 was said to be an "ideal curve." Necessarily inoculations will be more frequent.

In the case of tuberculous lymphatic glands and suppurating acne, which cases are probably the most numerous that one is called upon to treat, in which results such as depicted in Diagram 2 are aimed at, a slight subjective tenderness in the glands or an increase in the number of comedones respectively would not be of importance if lasting for not more than 24 hours, provided they were followed by a positive phase that lasted a week, more or less.

We now come to the consideration of the result of an inoculation as depicted in Diagram 3. With possibly one exception such a result is wholly bad. It is disastrous in acute general infections and it is at the best useless in chronic or acute local infections. The one exception is the case of a furuncle in such a condition of maturity that absorption without suppuration is unlikely to occur. In such a case it may be desirable to give such a dose of staphylococcus vaccine that a marked negative phase is produced during which rapid maturation with breaking down and subsequent evacuation of the abscess occurs.

The foregoing general considerations rest on the experience gained from the results of therapeutic inoculation as carried out at St. Mary's Hospital, where the majority of the inoculations are controlled by a varying number of opsonic indices. Nevertheless it is realised that in a vast number of cases the estimation of opsonic indices must be out of the question, and if it be a choice between therapeutic inoculation without opsonic indices or no inoculation at all, then therapeutic inoculation undertaken without indices most certainly is indicated. Moreover, we have now the accumulated experience of several years to generalise upon-an experience which takes cognisance of upwards of 15,000 opsonic estimations per year, carried out by the staff of the Department of Therapeutic Inoculation at St. Mary's Hospital. As the result of this experience the practice of vaccine therapy is becoming in certain directions more crystallised. Though we are more inclined to dispense with opsonic indices in the treatment of staphylococcal lesions than in any others, vaccine therapy without opsonic indices need not be confined to the treatment of such lesions, but may be applied to some cases of tuberculous, streptococcal, and gonococcal lesions as well as to the preventive inoculation of typhoid fever. For this purpose vaccines are prepared, standardised, and put up in bulbs of a little over one cubic centimetre each at St. Mary's Hospital and are obtainable through Messrs. Parke, Davis, and Co. These vaccines are precisely the same as are regularly used at St. Mary's. It is to be understood that the following statements are the outcome of the experience derived from the treatment of a large number of patients in hospital and in private, treatment which has almost invariably been controlled by frequent estimations of the opsonic index, and the writer in no way relinquishes the conviction that the best use of bacterial vaccines will always be made when the effects of each dose are ascertained by means of the opsonic index.

Having thus briefly considered the general effect of the inoculation of bacterial vaccines, it is advisable to pass to the consideration of applying the principles as above sketched out to the treatment of individual cases, or classes of case, and this can best be done under two main headings : (1) Diagnosis of the microbe at fault, its isolation and culture, and the preparation and standardisation of a vaccine; and (2) the estimation of the amount constituting a correct dose and the determination of the correct period that should elapse before re-inoculation.

1. For the complete conception of bacteriology indicated in the above subheading standard works on the subject must be consulted. Here it is only necessary to point out the elementary factors to be considered. From clinical experience many common lesions are always associated with certain micro-organisms and where such a comparative certainty exists it is sufficient to obtain the appropriate vaccine and proceed with inoculation. Such common diseases may be tabulated as follows, though no completeness is aimed at:-Staphylococci are mostly concerned in furunculosis and carbuncle, osteomyelitis, sycosis, and suppurating acne. Frequently in eczema and whitlow and occasionally in infective endocarditis and septicæmias. Streptococci are mostly concerned in erysipelas, puerperal septicæmia, septic endocarditis, lymphangitis, and most forms of cellulitis. The tubercle bacillus is concerned in many chronic local joint, glandular, and osseous swellings, and many skin lesions, besides phthisis, tuberculous peritonitis, and frequently in infections of the genito-urinary system. Thus in many lesions met with in everyday practice experience tells us, without precise bacteriological procedure, what is the probable causal microbe.

A few details as to the technique carried out where the diagnosis is not obvious may be indicated. Any discharge from the lesion can be directly examined by an ordinary smear preparation, stained either by a simple basic stain or by Gram's method. If more than one organism be present an endeavour must be made to determine which is the immediate pathogenic agent. Thus a mixture of staphylococcus and streptococcus usually leads one to the conclusion that the streptococcus is the causal agent of the lesion; should, however, an exhibition of streptococcus vaccine not be followed by amelioration one would then give staphylococcus vaccine or a mixture of both. Where, however, it is possible to have recourse to opsonic indices the question may be settled conclusively. If the index be normal to the staphylococcus isolated from the lesion and abnormal to the streptococcus the latter is certainly wholly or mostly responsible for the lesion. A septic endocarditis or other septicæmia can, in the absence of any obvious primary or secondary lesion, only be with certainty diagnosed by making a blood culture. To do this about 10 cubic centimetres of blood are taken with all aseptic precautions from a vein, usually and preferably the median basilic; about two cubic centimetres of blood are directly introduced into each of four tubes of sterile broth. One cubic centimetre may be introduced into a sterile flask of peptone water if typhoid fever paratyphoid or an infection due to a bacillus of the colon group, is suspected, and a portion may be spread on to the surface of an agar slope. If the organism be present in the blood it will in all probability grow in one of the media mentioned above. Subcultures are made and a vaccine is elaborated. Such measures, however, as a blood culture can only be carried out satisfactorily by a practised bacteriologist.

Apart from blood infections which may be diagnosed by means of blood cultures, many lesions may present themselves for treatment the cause of which is obscure. In such the opsonic index is of the greatest value, but it is beyond the 
scope of this article to do more than just indicate the procedure to be adopted.

As pointed out by Dr. John Freeman, the phenomenon of auto-inoculation is often observable where a circumscribed local infection is subjected to such treatment as to cause a temporary increase of circulation through the part. Such increased blood-flow may act in one of two ways, or in both ways at once. It may carry into the general circulation a greater or less amount of the products of the microbe, or of the microbe itself. If this be not excessive results comparable to those observed after an ordinary inoculation of bacterial vaccine occur; that is to say, a short negative phase followed by a positive phase and subsequent fall towards the pre-existing condition will occur and may be demonstrated if the opsonic indices to the organism in question be measured. Secondly, such increase of local circulation may cause an increased outflow of lymph, whereby a greater quantity of protective substances are carried into the infected areas and healing tends to result.

The phenomenon' of auto-inoculation may be made use of for the purpose of diagnosis. An obscure joint swelling may be so treated as to cause an increase of the local circulation when, if tubercle be present, a variation in the tuberculo-opsonic index will occur, or if gonococcus be the cause a variation will occur in the opsonic index to the gonococcus. An enlarged lymphatic gland may be carefully massaged and the blood examined before and after massage. If a variation of wide dimensions in the tuberculo-opsonic index occur tubercle is the cause of the swelling, or at least is present as a secondary infection. A variation to be diagnostic should extend over a distance at least equal to three-tenths of unity, unity being taken as the opsonic index of the normal healthy person. Thus a variation from 0.8 to 1.10 would be considered strong presumptive evidence of infection. The presumption is the greater if a number of opsonic indices when plotted out as a curve produce a curve without irregularities. Again, a high opsonic index is at all times more suggestive of infection than one below normal. It is to be pointed out that general and indiscriminate massage of infected joints or lymphatic glands is not advocated, each case being weighed on its own merits. At St. Mary's Hospital we constantly use Bier's methoc' of passive congestion as a means of producing a diagnostic autoinoculation. Cautious exercise of a previously immobile joint often gives valuable indications as to the nature and activity or otherwise of any infection, if opsonic indices before and after movement be taken.

The fringe only of the question of bacterial diagnosis is touched in the above remarks. The elucidation of any particular case must be left to the clinical observation of the practitioner, aided by such bacteriological technique as he may have at his command. Obscure infections must perforce be left to the bacteriologist and preferably one conversant with the technique of opsonic estimations. Such a question, for instance, as the diagnosis between tubercle and gonococcus may in some obscure cases baffle even an experienced bacteriologist, if he be not also an opsonist.

2. We now come to the consideration of the second subheading-viz, the estimation of the amount constituting a correct dose and the period that shouid elapse before reinoculation. While on the whole general principles only will be enunciated a few examples will be quoted, and for the sake of simplicity the cases to be considered will be divided into two main classes--viz.: (a) Those cases in which the exhibition of a correct dose of an appropriate vaccine will be followed by recognisable and obvious clinical signs without any delay. An example of this class of case is afforded by a furuncle and, indeed, staphylococcal lesions generally. (b) Those cases in which a dose of vaccine, be it ever so appropriate, is not followed immediately by recognisable and obvious clinical signs or, it may be, any sign whatever. An example of this class of case is often afforded by a tuberculous lymphatic gland in which even objective symptoms may often be absent even when the dosage is appropriate.

Now it follows that the treatment of these two classes of case must be approached from a totally different standpoint. In the first class, where obvious clinical signs may be expected to follow a dose of vaccine, it is possible to determine within a few days whether our dose has been appropriate or otherwise. Taking for example an incipient furuncle, we will follow out a typical case. A patient with a history of recurrent furunculosis presents himself with a commencing boil and with a view to aborting it one gives him 50 million staphylococci and gives directions that he attend again in four days. On his second attendance one of three indications may be present: he may be better, he may be in statu quo ante, or he may be worse. In case the furuncle was seen early enough he is likely to be better or in statu quo. If he be better one has evidence that the dose of vaccine was large enough to cause an elaboration of a sufficiency of protective substance to go towards an abortion of the lesion; a similar or slightly larger dose of vaccine would be indicated. Again, if there has been little or no change the indications are that though a rise in protective substances may have occurred in the circulating blood, it is either not high enough or the blood, though maybe rich in protective substances, is not having free access to the congested area comprised by the boil. A larger dose of vaccine is indicated and some measure designed to determine the blood to the part. Such a measure, for instance, is a series of fomentations. It is likely that these measures will result in the absorption of the congested fluids and a return to the normal condition on the part of the area of the boil. There will in that case be no further evidence immediately observable as to the accuracy of any dose, but to insure a more or less permanent immunity gradually increasing doses are indicated until a maximum of 400 or 500 millions be reached, and the doses may be given at first weekly and later at increasing intervals. On the other hand, should a furuncle be under consideration that is clearly about to suppurate, a larger dose, say 500 millions, may be immediately given. The resistance will be depressed temporarily and the boil will quickly ripen and discharge with or without incision as may seem best. A week after the first inoculation a small dose of 100 millions should be administered and doses rising by 50 millions or 100 millions a time should be given at first weekly and later at increasing periods until a maximum of 400 or 500 millions be reached.

Another example of a lesion that gives early evidence as to the appropriateness of the dose of vaccine is afforded by a case of suppurating acne. Here an appropriate dose is followed by a temporary exacerbation of the signs lasting for 24 hours, after which amelioration occurs. It the dose be too small an immediate amelioration is observed; such an amelioration, however, is but short-lived; should, on the other hand, the dose be excessive and a more or less profound negative phase be produced, the lesions get worse for several days and only slowly get back to the condition they were in at the period of inoculation. In some cases of acne, especially in females, large doses of staphylococci are ill borne at first, and it may be some months before a dose of 400 millions will be borne without a marked negative phase ensuing.

Many cases of chronic eczema are associated with a low resistance to staphylococcus together with an abnormal fluidity of the blood. Small doses, gradually increased, of staphylococci combined with the internal administration of lime, preferably calcium lactate, may be given, such procedure in most cases producing an immediate and marked effect. A case came under observation with a history of nearly 40 years' duration. Half a dozen doses of 250 million staphylococci at fortnightly intervals, together with the administration of half-drachm doses of calcium lactate every other day for three doses, with a weekly rest between each series of three doses, cleared up the case in three months. A slight relapse six months after was cleared up with two more doses of staphylococci and some more calcium lactate, and for 18 months there has been no recurrence.

The examples quoted above are of typical cases, but occasionally cases are met with in which the dose has to be extremely small, and where any dose is followed by a negative phase lasting more than a day or 36 hours, it may be concluded that the dose was too large, and a smaller one must be given on the next occasion. A case of furunculosis of some years' standing came under treatment, and it not being at first realised that the patient was peculiarly intolerant of the ordinary doses, the dose was rapidly raised, and it was not until on two separate occasions that serious negative phases, followed by crops of furuncles, were caused by doses of 250 millions that it became obvious that an intolerance existed. A complete cure followed, when a series of doses of 50 million staphylococci were given, a cure that 
has lasted without relapse for upwards of nine months and bids fair to be permanent.

Some cases of tuberculous infection will provide a certain amount of objective and possibly subjective signs by means of which guidance is afforded in dosage. Thus a tuberculous lymphatic gland may be rendered slightly tender to the touch, or even slightly painful by a certain dose. If such pain and tenderness last less than 24 hours it may be concluded that the dose was appropriate. Similarly a tuberculous joint infection may by increased pain and tenderness, with or without exercise, afford evidence as to the appropriateness of the previous dose.

In the case of streptococcus apart from acute general infections, the majority of the lesions to be treated will be local cellulitis, lymphangitis, and erysipelas, and secondary infections of tuberculous sinuses and lupus vulgaris. The local primary infections have to be approached with caution, and small doses only can be given. The clinical results are, however, so rapid as to afford very material evidence as to the appropriateness or otherwise of the antecedent inoculations. In the secondary streptococcal infections, notably those connected with tuberculous processes, the condition is as a rule, very chronic and sluggish. Larger doses of vaccine are borne without much negative phase, and indeed a slight negative phase may be not contra-indicated, as it may produce a flushing of the lesion with blood and increased exudation of lymph. The progress in these cases is, however, slow, and treatment may have to extend over several months. The secondary infection of lupus with streptococcus often obscures the clinical picture, and care must be exercised that a streptococcal infection be not mistaken for an exacerbation of the tuberculous process. Again, in gonococcal lesions, clinical signs afford valuable guidance. At St. Mary's Hospital the majority of our cases have been arthritic and in these an excessive dose is indicated by increased pain and stiffness with possibly a general malaise.

Some cases of urethritis have been treated with varying results. An autogenous vaccine, that is, one prepared from the patient's own organism, sometimes gives very good results. The negative phase is here indicated by an increase in the gleet, and if this lasts but one day a good result may be expected from the antecedent dose. With regard to gonococcus a marked difference occurs in the case of different vaccines. Thus a vaccine which we have been using for some months at St. Mary's Hospital gives good results in doses of from $2 \frac{1}{2}$ to 10 millions, other vaccines may require as a dose 40,50 , or possibly more millions. A recently isolated organism from a gleet gave no appreciable negative phase with an initial dose of 20 millions, and, indeed, only a slight fall towards normal was indicated opsonically, the index having been previously raised by the exhibition of a small dose of stock vaccine. By the fifth day, however, the resistance was nearly doubled and no organisms were found in the discharge which had previously abounded with gonococci. Examples might be multiplied indefinitely but those given above will suffice to indicate the lines to be proceeded upon in those cases in which clinical signs afford evidence as to the appropriateness or otherwise of each individual dose of vaccine.

The essential points in this class of case may be summarised thus. A dose of vaccine may be considered as appropriate when any negative phase, as indicated by objective or subjective signs, is only temporary and is followed by a longer or shorter period of improvement. Up to a certain point the period of improvement is proportional to the duration of the negative phase, but a point is soon reached when no improvement beyond the pre-existing condition is obtained. Beyond this point then a dose may be considered to be excessive. Conversely, if a short period of improvement with no signs of an intervening negative phase occur the succeeding dose of vaccine may be cautiously increased. As regards staphylococci the only exception to the above broadly stated rule would appear to be infective endocarditis and septicæmia, which are occasionally caused by staphylococcus. In such cases it would hardly be proposed that opsonic indices should be dispensed with, but were these latter out of the question it is obvious that extremely small doses should be given, though it might be advisable to repeat them with more than usual frequency, even to daily doses. Practically the only guide in such ca ses beyond the conditions of any local lesion that might be present would be the temperature. This, however, has been in some cases very valuable, indicating fairly accurately the state of resistance of the blood. Roughly, the temperature rises with a lowering of opsonic index, and vice versâ.

We now come to the consideration of the second class of case-namely, that class in which no objective signs immediately follow the exhibition of an appropriate dose of suitable vaccine. As an example of this class of case a tuberculous lymphatic gland was instanced. While it is true that in some cases a suitable dose of vaccine may be followed by slightly increased tenderness or even pain at the lesion, it is certain also that many, if not the majority, of these cases do not afford this evidence. Examples of negative phases that have been prolonged over several days as indicated by the opsonic index might be given in which no evidence as to increased tenderness or pain could be elicited. Again, in cases where two or more organisms are in question, the symptoms due to each several variety of microbe may not be obvious. In such it may be difficult to determine the value of any individual dose; it should be noted, however, that cases involving more than one organism are on the whole rare.

A tuberculous lymphatic gland may be taken as the most frequent lesion to be treated, in which clinical signs afford but little guidance. From week to week no great difference may be observable, and a long period may elapse even when resolution, as the result of appropriate doses of tuberculin, is occurring before the improvement is obvious, a period at least long enough to allow of several doses of tuberculin to be given. Hence it follows that there is no evidence as to which of several possibly varying doses of tuberculin given has been the correct one.

Not having the knowledge gained by constant estimations of the opsonic index recourse must be had to previous experience of similar cases. Before considering the application of this experience to particular cases it is advisable to differentiate between strictly local and more generalised cases of tuberculous infection. A much larger dose may be given in a strictly local case than in a more generalised case. Thus in the case of a robust adult presenting himself with a tuberculous lymphatic gland of the neck it would be good practice to give 1-10000th to 1-10000th of a milligramme of T.R. as an initial dose. If however, there were any involvement of the lung 1-30000th might be quite large enough a dose. The consideration of such lesions as pulmonary tuberculosis and tuberculous peritonitis must be superficial, how. ever; suffice it to say that any doses larger than 1-30000th to 1-20000th must be given with extreme caution. A negative phase of even a few hours' duration may give opportunity for disastrous activity of the tubercle bacilli in such, cases and with no evidence of the existence or otherwise of autoinoculation a dose which might be suitable one day might well be excessive the following day.

To return to such localised infections as tuberculous glands without any constitutional disturbance. It has been stated that in an adult an appropriate initial dose would be from 1-15000th to 1-10000th milligramme. Such a dose may be administered not more frequently than once every 10 days and may be raised gradually every third dose until 1-5000th to 1-4000th milligramme be reached. Usually it is unnecessary to proceed any higher and 1.4000th milligramme may be given for months with good effect. It is not suggested that every case can, however, stand such doses, but the exceptions will be few. Cases of lupus stand tuberculin well as a rule. Rarely have we occasion to give a dose as large as 1-2000th milligramme. It must be understood, however, that in the absence of the check afforded by opsonic indices any dose greater than 1-4000th milligramme of T.R. is dangerous, at least in the first six months of treatment. Cases of genito-urinary tuberculosis will not stand large doses of tuberculin as a rule, and 1-5000th should be regarded as a maximum. In children below the age of ten years the doses are smaller, though proportionately children take tuberculin well. A typical chart of the opsonic indices and doses of tuberculin is appended as an example (Fig. 2). The case was one treated in the ordinary routine of practice and the indices were not worked out with a view to the formation of a curve chart.

Note with regard to curve. - The dotted lines have been inserted to indicate the probable diagrammatic course of the resistance. It is to be noted that when any particular dose was ceasing to be the correct one the response was very rapid and short-lived. 
930 The Lancet,] MR. J. MATTHEWS: STOCK VACCINES AND BACTERIAL INFECTIONS. [SePt. 26, 1908.

From this it will be seen how small a dose was given in better example of chart than when only two estimations the first instance and how gradually it was increased. between each inoculation are undertaken, which latter That from a low average resistance a high average resist- number is usual in an average straightforward case. The ance was attained and maintained. That the average indications in such a case being to estimate the opsonic

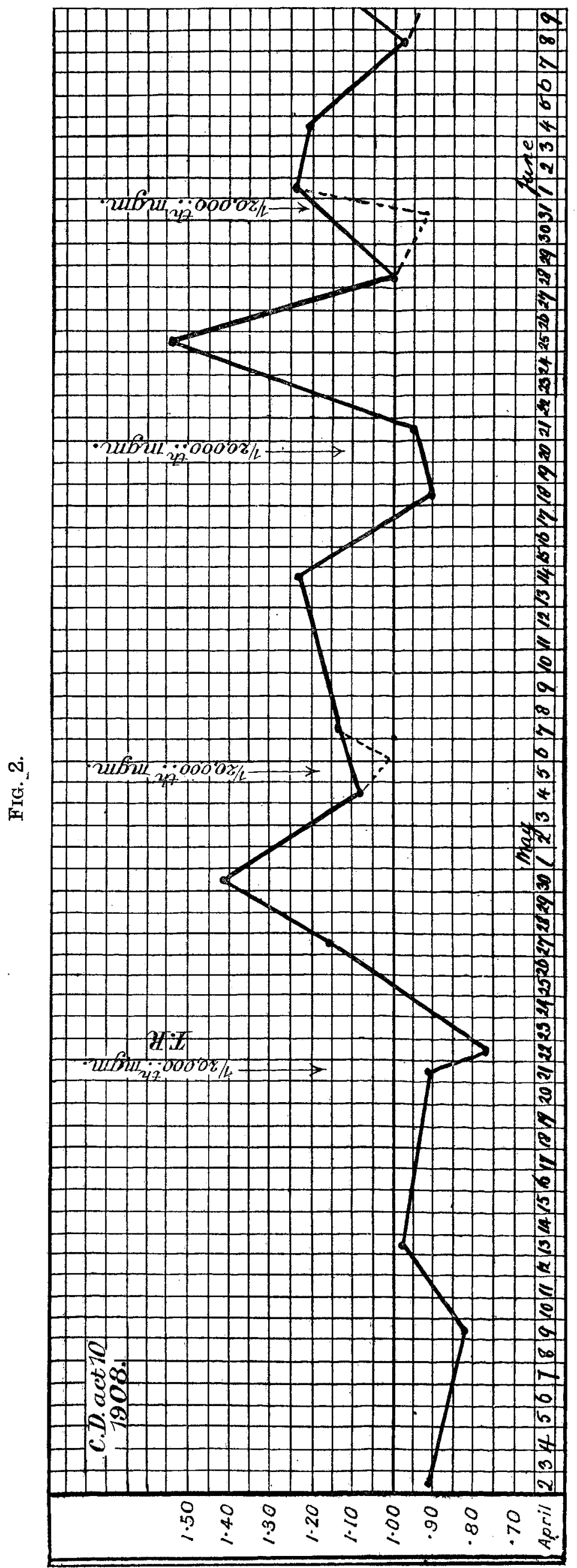

period above the normal line was much greater after the course of inoculations was started than the average period below the normal line.

This particular case is instanced because a generous number of opsonic indices was estimated and it affords a

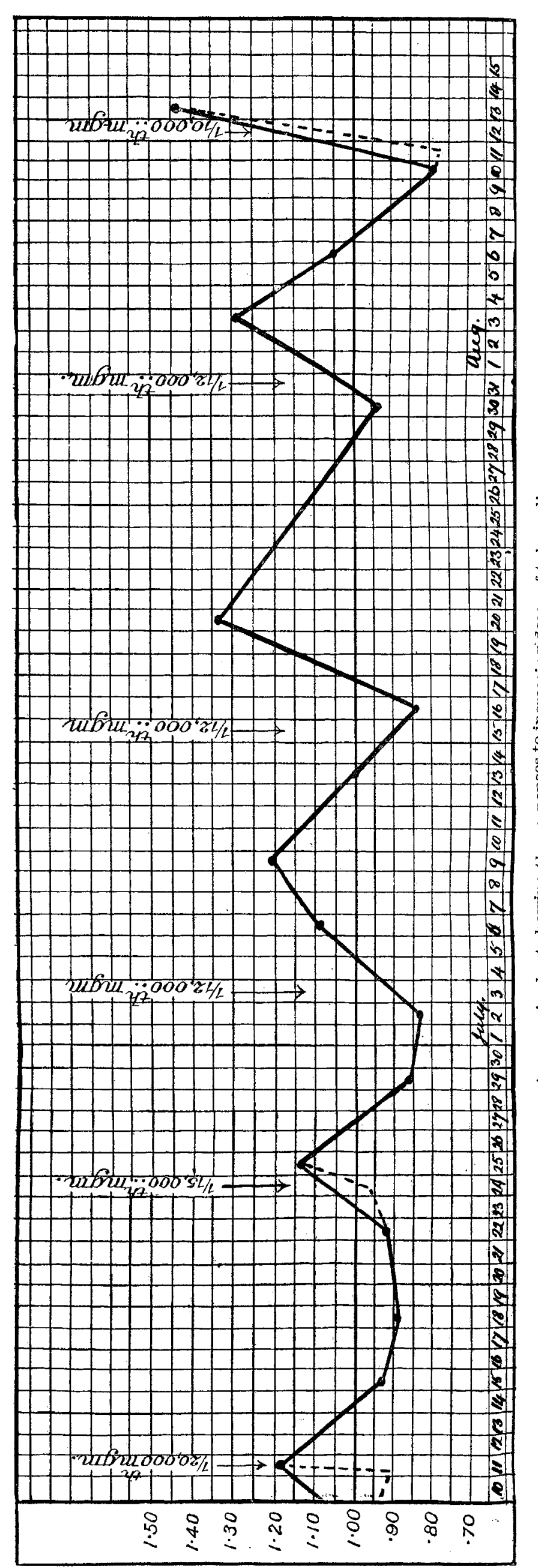

index 24 hours after inoculation to reveal a possibly excessive negative phase and on the seventh day or thereabouts to reveal the persistence or otherwise of the positive phase. If a high state of resistance be revealed inoculation is, of course, delayed for a few days. 
To summarise the treatment of cases in which no immediate indication as to the appropriate dose is afforded by clinical signs, such cases being generally tuberculous, it may be stated thus. In generalised tubercle, as phthisis and tuberculous peritonitis, larger doses than 1-30000th milligramme are to be given with the greatest caution, and in advanced cases such a dose even may be excessive. In strictly localised infections in adults the doses may range from 1-15000th to 1-4000th milligramme. In children with similar infections the doses may range from 1-25000th to 1-6000th, according to age and physique.

The indications as to dosage have designedly been slightly more dogmatic in the class of cases in which clinical evidence as to the appropriateness or otherwise of dosage is scanty or non-existent. Any hurry to increase the dose is deprecated, it being better to give several possibly nonmaximal doses than one large enough to produce a severe negative phase with a consequent set-back and the undoing of the good that may have pertained to many previous doses. The generalisation of the infection as opposed to strict localisation is the especial point to bear in mind. In the former small doses are to be insisted upon, for reasons given above.

In the preventive inoculation of typhoid fever previous experience can alone be relied on. Happily, this experience is extensive and the doses can be indicated with exactitude. 1000 million typhoid bacilli are administered and the patient is advised to rest, not necessarily in bed, for the rest of the day. A slight and varying amount of malaise, headache, and possibly joint pains will occur, but they usually pass off by the next day. Not less than ten days subsequent to the initial injection a dose of 2000 millions is given, and a similar sequence of symptoms may follow but is usually less marked than after the primary inoculation.

It is to be borne in mind that a high resistance in the circulating blood may not necessarily react in the direction of a cure. Unless the protective substances have free access to the area of infection the infective microbes have a highly beneficial nidus in which to multiply. Thus, the walls of a long-standing sinus may have a very poor bloodsupply by reason of pent-up discharge, due to obstruction to outflow or coagulation. In such a case the sinus may be flushed out with a hypertonic salt and citrate solution consisting of 4 per cent. of sodium chloride and 1 per cent. of sodium citrate in sterilised water. The hypertonicity of the solution promotes increased osmosis through the walls of the sinus and a more copious flow of lymph results; moreover, the citrate prevents coagulation and the increased flow of lymph through the sinus walls finds its way to the surface uncoagulated. The contents of a cavity, such as the peritoneal fluid in tuberculous peritonitis, the exudate in tuberculous pleurisy, or the contents of an abscess may be low in protective substances while the circulating blood contains an excess. Drainage of such a cavity is followed by the introduction of fluids rich in protective substances derived from the biood and the cavity, formerly a suitable breeding place for the infecting microbe, now becomes a highly unsuitable breeding place by reason of the increase in protective substances.

To meet the conditions as to dosage indicated in the foregoing remarks we prepare at St. Mary's various concentrations of vaccine. The different concentrations are so arranged that by quite simple calculations doses from the lowest to the highest figure indicated above may be given. The following is a list of vaccines prepared and issued :-

Tubercie raccine (I:R.). - Prepared from tubercle bacillary emulsion ; in two strengths-viz., 1-2000th and 1-5000th milligramme of dried comminuted tubercle bacilli per cubic centimetre.

Staphylocnecus vaccine.-Prepared from recently isolated virulent cultures of staphylococcus aureus and staphylococcus citreus; in three concentrations-viz., 100 millions, 200 millions, and 500 millions of cocci per cubic centimetre.

Streptococcus vaccine.-Prepared from recently isolated cultures of streptococcus obtained from cases of erysipelas; in three concentrations-viz., 5 millions, 10 millions, and 50 millions per cubic centimetre.

Gonococcus vacine. - Prepared from cultures of proved immunising power; in two concentrations-viz., 5 millions and 10 millions of cocci per cubic centimetre.

Typhoid vacoine.-Prepared from a culture that has been frequently tested as to its immunising power; in two concentrations-viz., 1000 millions and 2000 millions of bacilli per cubic centimetre.

There is a probability that a vaccine of micrococcus neoformans will shortly be issued, as evidence is accruing of the beneficial results as regards lessening of pain and retardation of growth and secondary deposits in some kinds of cancer. It will probably be issued in one concentrationviz., 50 millions per cubic centimetre. 15 millions may be regarded as an appropriate initial dose and 25 millions as an average dose.

The T.R. is prepared from Koch's bacillary emulsion obtained from Meister Lucius and Brüning. It is to be noted that the dilutions are made and standardised on the assumption that one cubic centimetre of the product as issued by Meister Lucius and Brüning contains two, and not ten, milligrammes of dried comminuted tubercle bacilli. The stock staphylococcal vaccine, as issued to the profession, is used at St. Mary's for practically all staphylococcal lesions. Very rarely is it necessary to make a speclal autogenous vaccine. The stock streptococcal vaccine is used initially in acute cases. As soon as the infecting streptococcus is isolated a special vaccine is almost invariably prepared, these being far more specific. Rarely is it possible to prepare special gonococcal vaccines, so the stock vaccine has usually to be relied on.

Practically all other "lesions, more especially those due to bacillus coli communis, are treated with specially prepared autogenous vaccines, and with regard to the latter there does not appear to be the slightest likelihood of a stock vaccine proving of any use in more than a small percentage of infections due to the colon bacillus.

\section{VACCINES IN GENERAL PRACTICE.}

\author{
BY LACHLAN GRANT, M.D., O.M. EdIN.; \\ T. H. CAMPBELL, M.B., OH.B. GLASG.; \\ AND
}

W. D. ANDERson, M.A., M.B. Cantab., M.R.C.S. EnG.

THE following cases having resisted the older forms of treatment it was decided to try the effect of vaccine therapy, and in these notes we will give a brief history of the cases, the modus operandi employed, and the results obtained.

CASE 1.-A man, aged 35 years, with a tuberculous history, consulted us in September, 1907, regarding a large swelling of two months' duration, on the left side of the neck, which extended from the angle of the jaw almost to the manubrium sterni. The case was one of advanced tuberculosis of the cervical glands which were evidently in a state of caseation and liquefaction. A few days later an incision was made into the dependent part of the swelling and the fluid contents were evacuated. The swelling subsided considerably and during the following three weeks the disease seemed to be held in abeyance. The improvement, however, was only temporary and the swelling advancing downwards another incision became necessary. In the course of another month, owing to the disease having spread over the manubrium, it was found expedient to make a third incision. The wounds were throughout treated antiseptically and all precautions were taken against the possible occurrence of mixed infection. Absolute rest in bed was enjoined, the patient's diet was regulated, and the best hygienic measures possible were adopted. This proved of little avail. The wounds remained open, sinuses discharging tuberculous débris. Indeed, an extension along the deep fascia into the mediastinum was feared. The affected part, after remaining unchanged for about three months, was subjected to the $x$ rays. Irradiation was given on three separate occasions at intervals of one week, the dose on each occasion being 15 minutes of a soft tube with 0.35 milliampères passing and the anticathode 12 inches from the diseased tissue. After each irradiation a thin friable cicatrix formed over the wounds, but it in variably broke down again after a few days. The patient had been under our care for seven months and we now decided on a vaccine treatment.

On May 11th, 1908, an estimation of the patient's opsonic index for tubercle bacillus was made, and the index was found to be $0 \cdot 7$. On the same day $1-5000$ cubic centimetre of T.R. was injected into the infrascapular region. This 